\title{
EXISTENCE OF SOLUTIONS AND CONTROLLABILITY FOR IMPULSIVE FRACTIONAL ORDER DAMPED SYSTEMS
}

\author{
ZHENHAI LIU AND XUEMEI LI \\ Communicated by $\mathrm{Da} \mathrm{Xu}$
}

\begin{abstract}
In this paper, we are concerned with the controllability of linear and nonlinear Caputo impulsive fractional order damped systems in Banach spaces. Our main purpose is to establish some necessary and sufficient conditions for controllability for this kind of impulsive control system by using Mittag-Leffler matrix functions and the Schauder fixed point theorem.
\end{abstract}

1. Introduction. Control theory is an area of application-oriented mathematics which deals with basic principles underlying the analysis and design of control systems. It is well known that the study of controllability plays an important role in control theory and engineering. Although most dynamical systems are analyzed in either the continuous- or discrete-time domain, many real systems in physics, chemistry, biology, engineering, and information science, may experience abrupt changes at certain instances during continuous dynamical processes. These kinds of impulsive behaviors can be modeled by impulsive systems. Instantaneous impulsive fractional differential equations are used to describe some practical dynamical systems including evolutionary processes which are characterized by abrupt changes of the state in certain instances. The theory of instantaneous impulsive fractional differential equations has found its extensive applications in realistic mathematical models and has emerged as an important area of investigation in recent years. The study of impulsive control

2010 AMS Mathematics subject classification. Primary 34K37, 93B05.

Keywords and phrases. Controllability, impulsive fractional differential equations, Mittag-Leffler function, fixed point theorem.

Project supported by NNSF of China, grant No. 11671101 and Special Funds of Guangxi Distinguished Experts Construction Engineering.

Received by the editors on November 8, 2015, and in revised form on May 25, 2016. 
systems has received increasing interest and has had rapid development in the past few years, since dynamical systems with impulsive effects have great importance in applied sciences (see, for example, $[3,4,6,8,9,10,11,12,16,17,18,20,21,22,23,24,25,26]$ and the references therein).

In this paper, we study controllability results for the following fractional order damped impulsive linear control systems:

$$
\begin{cases}{ }^{c} D_{t}^{\alpha} x(t)=A^{c} D_{t}^{\beta} x(t)+B u(t) & t \in J^{\prime} \\ \Delta x\left(t_{i}\right):=I_{i}\left(x\left(t_{i}^{-}\right)\right), \Delta x^{\prime}\left(t_{i}\right):=J_{i}\left(x^{\prime}\left(t_{i}^{-}\right)\right) & i=1,2, \ldots, k \\ x(0)=x_{0}, x^{\prime}(0)=x_{0}^{\prime} & \end{cases}
$$

and the following fractional order damped impulsive nonlinear control systems:

$$
\begin{cases}{ }^{c} D_{t}^{\alpha} x(t)=A^{c} D_{t}^{\beta} x(t)+B u(t)+f(t, x(t)) & t \in J^{\prime} \\ \Delta x\left(t_{i}\right):=I_{i}\left(x\left(t_{i}^{-}\right)\right), \Delta x^{\prime}\left(t_{i}\right):=J_{i}\left(x^{\prime}\left(t_{i}^{-}\right)\right) & i=1,2, \ldots, k \\ x(0)=x_{0}, x^{\prime}(0)=x_{0}^{\prime} & \end{cases}
$$

where $0<\beta \leq 1<\alpha \leq 2,{ }^{c} D_{t}^{\alpha}$ and ${ }^{c} D_{t}^{\beta}$ denote the Caputo fractional derivatives of orders $\alpha$ and $\beta$ with lower limit zero, respectively. $A$ and $B$ are $n \times n$ and $n \times m$ matrices, respectively.

$$
J=[0, T], \quad J^{\prime}=J-\left\{t_{1}, t_{2}, \cdots, t_{k}\right\}
$$

and

$$
0=t_{0}<t_{1}<t_{2}<\cdots<t_{i}<\cdots<t_{k}<t_{k+1}=T<\infty,
$$

$f: J \times \mathbb{R}^{n} \rightarrow \mathbb{R}^{n}, I_{i}, J_{i}: \mathbb{R}^{n} \rightarrow \mathbb{R}^{n}, i=1,2, \ldots, k . x(t) \in \mathbb{R}^{n}$ is the state variable. $u(t) \in \mathbb{R}^{m}$ is the control variable. $\Delta x\left(t_{i}\right):=x\left(t_{i}^{+}\right)-x\left(t_{i}^{-}\right)$, where $x\left(t_{i}^{+}\right)=\lim _{\epsilon \rightarrow 0^{+}} x\left(t_{i}+\epsilon\right)$ and $x\left(t_{i}^{-}\right)=\lim _{\epsilon \rightarrow 0^{-}} x\left(t_{i}+\epsilon\right)$ represent the right and left limits of $x(t)$ at $t=t_{i}$, respectively. $\Delta x^{\prime}\left(t_{i}\right)$ is similarly defined.

The fractional order damped control systems without impulse effect in finite-dimensional spaces above were considered by Balachandran, Govindaraj, Reiver and Trujillo [2]. They studied the controllability of linear and nonlinear fractional order damped systems. In this paper, we deal with the controllability of linear and nonlinear Caputo impulsive fractional order damped systems. Our main purpose is to establish 
some necessary and sufficient conditions of controllability for this type of impulsive control system by using Mittag-Leffler matrix functions and the Schauder fixed point theorem.

This paper is organized as follows. In Section 2, we give some notation and recall some preliminary material. In Section 3, we give the solution expression of impulsive fractional systems. In Section 4, we study the controllability of systems (1.1) and (1.2).

2. Preliminaries. In this section, we introduce some basic definitions and preliminaries which are used throughout this paper. Let $\mathbb{R}^{n}$ be the $n$-dimensional Euclidean space, $\mathbb{R}_{+}=[0, \infty)$. Let $C\left(J, \mathbb{R}^{n}\right)$ be the Banach space of all continuous functions from $J$ into $\mathbb{R}^{n}$ with the norm

$$
\|u\|_{c}:=\sup \left\{\|u(t)\|_{\mathbb{R}^{n}}: t \in J\right\} \quad \text { for } u \in C\left(J, \mathbb{R}^{n}\right) .
$$

For brevity, $\|u(t)\|_{\mathbb{R}^{n}}$ is taken as $\|u(t)\|$. We also introduce the spaces:

$$
\begin{array}{r}
P C\left(J, \mathbb{R}^{n}\right)=\left\{u: J \rightarrow \mathbb{R}^{n} \mid u \in C\left(\left(t_{i}, t_{i+1}\right], \mathbb{R}^{n}\right), u\left(t_{i}^{-}\right), u\left(t_{i}^{+}\right)\right. \text {exist } \\
\text { and } \left.u\left(t_{i}^{-}\right)=u\left(t_{i}\right), i=0,1,2, \ldots, k\right\},
\end{array}
$$

with the norms

$$
\begin{aligned}
\|u\|_{P C} & :=\sup \{\|u(t)\|: t \in J\} \\
P C^{1}\left(J, \mathbb{R}^{n}\right) & =\left\{u \in P C\left(J, \mathbb{R}^{n}\right): u^{\prime} \in P C\left(J, \mathbb{R}^{n}\right)\right\}
\end{aligned}
$$

and

$$
\|u\|_{P C^{1}}:=\max \left\{\|u\|_{P C},\left\|u^{\prime}\right\|_{P C}\right\}
$$

Obviously, $P C\left(J, \mathbb{R}^{n}\right)$ and $P C^{1}\left(J, \mathbb{R}^{n}\right)$ are Banach spaces. The Banach space of all Lebesgue $p$-integrable functions from $J$ into $\mathbb{R}^{n}$ with

$$
\|f\|_{L^{p}}:=\left(\int_{J}\|f(t)\|^{p} d t\right)^{1 / p}
$$

are denoted by $L^{p}\left(J, \mathbb{R}^{n}\right)$.

Firstly, let us recall the following basic definitions from fractional calculus. For more details, see $[7,15]$. 
Definition 2.1. The fractional integral of order $\alpha$ with lower limit 0 for a function $f:[0, \infty) \rightarrow \mathbb{R}$ is defined as

$$
I_{t}^{\alpha} f(t)=\frac{1}{\Gamma(\alpha)} \int_{0}^{t}(t-s)^{\alpha-1} f(s) d s, \quad \alpha>0, t>0,
$$

provided the right-hand side is pointwise defined on $[0, \infty)$, where $\Gamma$ is the Gamma function.

Definition 2.2. The Caputo derivative of order $\alpha$ with lower limit 0 for a function $f:[0, \infty) \rightarrow \mathbb{R}$ can be written as:

$$
\begin{aligned}
{ }^{c} D_{t}^{\alpha} f(t)= & \frac{1}{\Gamma(n-\alpha)} \int_{0}^{t}(t-s)^{n-\alpha-1} f^{(n)}(s) d t, \\
& 0<n-1<\alpha \leq n .
\end{aligned}
$$

Its Laplace transform is defined by

$$
L\left\{{ }^{c} D_{t}^{\alpha} f(t)\right\}(s)=s^{\alpha} F(s)-\sum_{k=0}^{n-1} f^{(k)}\left(0^{+}\right) s^{\alpha-1-k} .
$$

In particular, if $0<\alpha \leq 1$, then

$$
L\left\{{ }^{c} D_{t}^{\alpha} f(t)\right\}(s)=s^{\alpha} F(s)-f\left(0^{+}\right) s^{\alpha-1},
$$

and, if $1<\alpha \leq 2$, then

$$
L\left\{{ }^{c} D_{t}^{\alpha} f(t)\right\}(s)=s^{\alpha} F(s)-f\left(0^{+}\right) s^{\alpha-1}-f^{\prime}\left(0^{+}\right) s^{\alpha-2} .
$$

Next, let us proceed to the definition and properties of the MittagLeffler function which can be found in $[7,13,14,15]$.

Definition 2.3. The two-parameter Mittag-Leffler function is defined by:

$$
E_{\alpha, \beta}(Z)=\sum_{k=0}^{\infty} \frac{Z^{k}}{\Gamma(\alpha k+\beta)}, \quad \alpha>0, \beta>0,
$$

where $Z \in \mathbb{C}$. The Laplace transform of the Mittag-Leffler function is:

$$
L\left\{t^{\beta-1} E_{\alpha, \beta}\left( \pm a t^{\alpha}\right)\right\}(s)=\frac{s^{\alpha-\beta}}{s^{\alpha} \mp a}, \quad \operatorname{Re}(s)>|a|^{1 / \alpha} .
$$


In particular, for $\beta=1$,

$$
E_{\alpha, 1}(Z)=E_{\alpha}(Z)=\sum_{k=0}^{\infty} \frac{Z^{k}}{\Gamma(\alpha k+1)}, \quad Z \in \mathbb{C} .
$$

Remark 2.4. Let $A \in \mathbb{C}^{n \times n}$. The matrix extension of the aforementioned Mittag-Leffler function has the following representation:

$$
E_{\alpha, \beta}\left(A t^{\alpha}\right)=\sum_{k=0}^{\infty} \frac{A^{k} t^{k \alpha}}{\Gamma(\alpha k+\beta)}, \quad \alpha>0, \beta>0
$$

and

$$
L\left\{t^{\beta-1} E_{\alpha, \beta}\left(A t^{\alpha}\right)\right\}(s)=\left(s^{\alpha} I-A\right)^{-1} s^{\alpha-\beta}, \quad \operatorname{Re}(s)>\|A\|^{1 / \alpha} .
$$

In order to define the solution of systems (1.1) and (1.2), we need:

Lemma 2.5 (cf. [9]). If $f \in P C^{n}(J, \mathbb{R}), n-1<\alpha<n$, then we have the following general expression:

$$
\begin{aligned}
& I_{t}^{\alpha c} D_{t}^{\alpha} f(t) \\
& = \begin{cases}f(t)-\sum_{j=0}^{n-1} \frac{f^{(j)}(0)}{j !} t^{j} & t \in\left[0, t_{1}\right], \\
f(t)-\sum_{j=0}^{n-1} \frac{f^{(j)}(0)}{j !} t^{j} & \\
\quad-\sum_{m=1}^{i} \sum_{j=0}^{n-1} \frac{\Delta f^{(j)}\left(t_{m}\right)}{j !}\left(t-t_{m}\right)^{j} & t \in\left(t_{i}, t_{i+1}\right], i=1,2, \ldots, k .\end{cases}
\end{aligned}
$$

3. The solution of impulsive fractional systems. In the present section, we turn our attention to the solution formula of the impulsive fractional control systems. Now, according to Lemma 2.5, we have the following.

Theorem 3.1. Let $\alpha \in(1,2], \beta \in(0,1], B \in \mathbb{R}^{n \times m}$ and $u \in$ $L^{1 / p}\left(J, \mathbb{R}^{m}\right), p \in(0, \alpha-1)$, if $x \in P C^{1}\left(J, \mathbb{R}^{n}\right)$, and $x$ is a solution 
of the following problem:

$$
\begin{cases}{ }^{c} D_{t}^{\alpha} x(t)=A^{c} D_{t}^{\beta} x(t)+B u(t) & t \in J^{\prime} \\ \Delta x\left(t_{i}\right)=I_{i}\left(x\left(t_{i}^{-}\right)\right), \Delta x^{\prime}\left(t_{i}\right)=J_{i}\left(x^{\prime}\left(t_{i}^{-}\right)\right) & i=1,2, \ldots, k, \\ x(0)=x_{0}, x^{\prime}(0)=x_{0}^{\prime} & \end{cases}
$$

Then, $x$ satisfies the following equation:

$$
x(t)=\left\{\begin{array}{l}
E_{\alpha-\beta}\left(A t^{\alpha-\beta}\right) x_{0}+t E_{\alpha-\beta, 2}\left(A t^{\alpha-\beta}\right) x_{0}^{\prime} \\
\quad-A t^{\alpha-\beta} E_{\alpha-\beta, \alpha-\beta+1}\left(A t^{\alpha-\beta}\right) x_{0} \\
\quad+\int_{0}^{t}(t-s)^{\alpha-1} E_{\alpha-\beta, \alpha}\left(A(t-s)^{\alpha-\beta}\right) B u(s) d s, \quad t \in\left[0, t_{1}\right], \\
E_{\alpha-\beta}\left(A t^{\alpha-\beta}\right) x_{0}-A t^{\alpha-\beta} E_{\alpha-\beta, \alpha-\beta+1}\left(A t^{\alpha-\beta}\right) x_{0} \\
\quad+t E_{\alpha-\beta, 2}\left(A t^{\alpha-\beta}\right) x_{0}^{\prime}+\sum_{j=1}^{i} E_{\alpha-\beta}\left(A\left(t-t_{j}\right)^{\alpha-\beta}\right) I_{j}\left(x\left(t_{j}^{-}\right)\right) \\
\quad-A \sum_{j=1}^{i}\left(t-t_{j}\right)^{\alpha-\beta} E_{\alpha-\beta, \alpha-\beta+1}\left(A\left(t-t_{j}\right)^{\alpha-\beta}\right) I_{j}\left(x\left(t_{j}^{-}\right)\right) \\
\quad+\sum_{j=1}^{i}\left(t-t_{j}\right) E_{\alpha-\beta, 2}\left(A\left(t-t_{j}\right)^{\alpha-\beta}\right) J_{j}\left(x^{\prime}\left(t_{j}^{-}\right)\right) \\
\quad+\int_{0}^{t}(t-s)^{\alpha-1} E_{\alpha-\beta, \alpha}\left(A(t-s)^{\alpha-\beta}\right) B u(s) d s, \\
\quad t \in\left(t_{i}, t_{i+1}\right], \quad i=1,2, \ldots, k .
\end{array}\right.
$$

Proof. We observe that $x(\cdot)$ can be decomposed to $v(\cdot)+w(\cdot)$, where $v$ is the continuous solution for

$$
\left\{\begin{array}{l}
{ }^{c} D_{t}^{\alpha} v(t)=A^{c} D_{t}^{\beta} v(t)+B u(t) \quad t \in J \\
v(0)=x_{0}, v^{\prime}(0)=x_{0}^{\prime}
\end{array}\right.
$$

and $w$ is the solution for

$$
\begin{cases}{ }^{c} D_{t}^{\alpha} w(t)=A^{c} D_{t}^{\beta} w(t) & t \in J^{\prime} \\ \Delta w\left(t_{i}\right)=I_{i}\left(x\left(t_{i}^{-}\right)\right), \Delta w^{\prime}\left(t_{i}\right)=J_{i}\left(x^{\prime}\left(t_{i}^{-}\right)\right) & i=1,2, \ldots, k, \\ w(0)=0, w^{\prime}(0)=0 . & \end{cases}
$$

Firstly, by [2], we get that the solution of equation (3.3) is given by:

$$
\begin{aligned}
v(t)= & E_{\alpha-\beta}\left(A t^{\alpha-\beta}\right) x_{0}-A t^{\alpha-\beta} E_{\alpha-\beta, \alpha-\beta+1}\left(A t^{\alpha-\beta}\right) x_{0} \\
& +t E_{\alpha-\beta, 2}\left(A t^{\alpha-\beta}\right) x_{0}^{\prime}
\end{aligned}
$$




$$
+\int_{0}^{t}(t-s)^{\alpha-1} E_{\alpha-\beta, \alpha}\left(A(t-s)^{\alpha-\beta}\right) B u(s) d s, \quad t \in J .
$$

Next, we calculate the solution of equation (3.4).

In order to do this, we apply the Riemann-Liouville fractional integral operator on both sides of the equation (3.4), to obtain

$$
I_{t}^{\alpha c} D_{t}^{\alpha} w(t)=A I_{t}^{\alpha c} D_{t}^{\beta} w(t)=A I_{t}^{\alpha-\beta}\left(I_{t}^{\beta}{ }^{c} D_{t}^{\beta} w(t)\right) .
$$

If $t \in\left[0, t_{1}\right]$, by Lemma 2.5, we have

$$
w(t)-w(0)-w^{\prime}(0) t=A I_{t}^{\alpha-\beta} w(t)-A I_{t}^{\alpha-\beta} w(0) .
$$

Since $w(0)=0, w^{\prime}(0)=0$, then

$$
w(t)=\frac{A}{\Gamma(\alpha-\beta)} \int_{0}^{t}(t-s)^{\alpha-\beta-1} w(s) d s .
$$

If $t \in\left(t_{i}, t_{i+1}\right], i=1,2, \ldots, k$, again by Lemma 2.5 , we have

$$
\begin{aligned}
w(t)-w(0)-w^{\prime}(0) t-\sum_{j=1}^{i} \Delta & w\left(t_{j}\right)-\sum_{j=1}^{i} \Delta w^{\prime}\left(t_{j}\right)\left(t-t_{j}\right) \\
& =A I_{t}^{\alpha-\beta}\left[w(t)-w(0)-\sum_{j=1}^{i} \Delta w\left(t_{j}\right)\right] .
\end{aligned}
$$

It follows from equation (3.4) that

$$
\begin{aligned}
w(t)= & \sum_{j=1}^{i} \Delta w\left(t_{j}\right)+\sum_{j=1}^{i} \Delta w^{\prime}\left(t_{j}\right)\left(t-t_{j}\right)+A I_{t}^{\alpha-\beta} w(t) \\
& -\frac{A\left(t-t_{j}\right)^{\alpha-\beta}}{\Gamma(\alpha-\beta+1)} \sum_{j=1}^{i} \Delta w\left(t_{j}\right) \\
= & \sum_{j=1}^{i} I_{j}\left(x\left(t_{j}^{-}\right)\right)-\frac{A\left(t-t_{j}\right)^{\alpha-\beta}}{\Gamma(\alpha-\beta+1)} \sum_{j=1}^{i} I_{i}\left(x\left(t_{j}^{-}\right)\right) \\
& +\sum_{j=1}^{i} J_{j}\left(x^{\prime}\left(t_{j}^{-}\right)\right)\left(t-t_{j}\right)
\end{aligned}
$$




$$
+\frac{A}{\Gamma(\alpha-\beta)} \int_{0}^{t}(t-s)^{\alpha-\beta-1} w(s) d s .
$$

Equations (3.5)and (3.6) can be rewritten as

$$
\begin{aligned}
w(t)= & \sum_{j=1}^{i} I_{j}\left(x\left(t_{j}^{-}\right)\right) \chi_{j}(t)+\sum_{j=1}^{i} J_{j}\left(x^{\prime}\left(t_{j}^{-}\right)\right) \chi_{j}(t)\left(t-t_{j}\right) \\
& -\frac{A\left(t-t_{j}\right)^{\alpha-\beta}}{\Gamma(\alpha-\beta+1)} \sum_{j=1}^{i} I_{j}\left(x\left(t_{j}^{-}\right)\right) \chi_{j}(t) \\
& +\frac{A}{\Gamma(\alpha-\beta)} \int_{0}^{t}(t-s)^{\alpha-\beta-1} w(s) d s, \quad t \in\left(t_{i}, t_{i+1}\right]
\end{aligned}
$$

where

$$
\chi_{j}(t)= \begin{cases}0 & t \leq t_{j} \\ 1 & t>t_{j}\end{cases}
$$

Let $\lambda>0$. We adopt the idea in [25] and apply the Laplace transformation to (3.7). Then

$$
\begin{aligned}
W(\lambda)= & \sum_{j=1}^{i} \frac{e^{-\lambda t_{j}}}{\lambda} I_{j}\left(x\left(t_{j}^{-}\right)\right)+\sum_{j=1}^{i} \frac{e^{-\lambda t_{j}}}{\lambda^{2}} J_{j}\left(x^{\prime}\left(t_{j}^{-}\right)\right) \\
& -\sum_{j=1}^{i} \frac{A e^{-\lambda t_{j}}}{\lambda^{\alpha-\beta+1}} I_{j}\left(x\left(t_{j}^{-}\right)\right)+\frac{A}{\lambda^{\alpha-\beta}} W(\lambda),
\end{aligned}
$$

which implies

$$
\begin{aligned}
W(\lambda)= & \sum_{j=1}^{i} \lambda^{\alpha-\beta-1}\left(\lambda^{\alpha-\beta} I-A\right)^{-1} e^{-\lambda t_{j}} I_{j}\left(x\left(t_{j}^{-}\right)\right) \\
& +\sum_{j=1}^{i} \lambda^{\alpha-\beta-2}\left(\lambda^{\alpha-\beta} I-A\right)^{-1} e^{-\lambda t_{j}} J_{j}\left(x^{\prime}\left(t_{j}^{-}\right)\right) \\
& -\sum_{j=1}^{i} \lambda^{-1} A\left(\lambda^{\alpha-\beta} I-A\right)^{-1} e^{-\lambda t_{j}} I_{j}\left(x\left(t_{j}^{-}\right)\right) .
\end{aligned}
$$

Note that the Laplace transform of $t^{\beta-1} E_{\alpha, \beta}\left(A t^{\alpha}\right)$ is $\lambda^{\alpha-\beta}\left(\lambda^{\alpha-\beta} I-\right.$ $A)^{-1}$. Thus, we can calculate the solution of (3.4) as 


$$
\begin{aligned}
w(t)= & \sum_{j=1}^{i} \chi_{j}(t) E_{\alpha-\beta}\left(A\left(t-t_{j}\right)^{\alpha-\beta}\right) I_{j}\left(x\left(t_{j}^{-}\right)\right) \\
& +\sum_{j=1}^{i} \chi_{j}(t)\left(t-t_{j}\right) E_{\alpha-\beta, 2}\left(A\left(t-t_{j}^{\alpha-\beta}\right) J_{j}\left(x^{\prime}\left(t_{j}^{-}\right)\right)\right. \\
& -A \sum_{j=1}^{i} \chi_{j}(t)\left(t-t_{j}\right)^{\alpha-\beta} E_{\alpha-\beta, \alpha-\beta+1}\left(A\left(t-t_{j}\right)^{\alpha-\beta}\right) I_{j}\left(x\left(t_{j}^{-}\right)\right) .
\end{aligned}
$$

By the above, the solution of equation (3.1) can be given by:

$$
\begin{aligned}
x(t)= & E_{\alpha-\beta}\left(A t^{\alpha-\beta}\right) x_{0}-A t^{\alpha-\beta} E_{\alpha-\beta, \alpha-\beta+1}\left(A t^{\alpha-\beta}\right) x_{0} \\
& +t E_{\alpha-\beta, 2}\left(A t^{\alpha-\beta}\right) x_{0}^{\prime}+\sum_{j=1}^{i} \chi_{j}(t) E_{\alpha-\beta}\left(A\left(t-t_{j}\right)^{\alpha-\beta}\right) I_{j}\left(x\left(t_{j}^{-}\right)\right) \\
& +\sum_{j=1}^{i} \chi_{j}(t)\left(t-t_{j}\right) E_{\alpha-\beta, 2}\left(A\left(t-t_{j}^{\alpha-\beta}\right) J_{j}\left(x^{\prime}\left(t_{j}^{-}\right)\right)\right. \\
& -A \sum_{j=1}^{i} \chi_{j}(t)\left(t-t_{j}\right)^{\alpha-\beta} E_{\alpha-\beta, \alpha-\beta+1}\left(A\left(t-t_{j}\right)^{\alpha-\beta}\right) I_{j}\left(x\left(t_{j}^{-}\right)\right) \\
& +\int_{0}^{t}(t-s)^{\alpha-1} E_{\alpha-\beta, \alpha}\left(A(t-s)^{\alpha-\beta}\right) B u(s) d s, \quad t \in\left(t_{i}, t_{i+1}\right],
\end{aligned}
$$

that is,

$$
x(t)=\left\{\begin{aligned}
E_{\alpha-\beta}\left(A t^{\alpha-\beta}\right) x_{0}-A t^{\alpha-\beta} E_{\alpha-\beta, \alpha-\beta+1}\left(A t^{\alpha-\beta}\right) x_{0}+t E_{\alpha-\beta, 2}\left(A t^{\alpha-\beta}\right) x_{0}^{\prime} \\
\quad+\int_{0}^{t}(t-s)^{\alpha-1} E_{\alpha-\beta, \alpha}\left(A(t-s)^{\alpha-\beta}\right) B u(s) d s, t \in\left[0, t_{1}\right] \\
E_{\alpha-\beta}\left(A t^{\alpha-\beta}\right) x_{0}-A t^{\alpha-\beta} E_{\alpha-\beta, \alpha-\beta+1}\left(A t^{\alpha-\beta}\right) x_{0}+t E_{\alpha-\beta, 2}\left(A t^{\alpha-\beta}\right) x_{0}^{\prime} \\
\quad+\sum_{j=1}^{i} E_{\alpha-\beta}\left(A\left(t-t_{j}\right)^{\alpha-\beta}\right) I_{j}\left(x\left(t_{j}^{-}\right)\right) \\
\quad+\sum_{j=1}^{i}\left(t-t_{j}\right) E_{\alpha-\beta, 2}\left(A\left(t-t_{j}\right)^{\alpha-\beta}\right) J_{j}\left(x^{\prime}\left(t_{j}^{-}\right)\right) \\
\quad-A \sum_{j=1}^{i}\left(t-t_{j}\right)^{\alpha-\beta} E_{\alpha-\beta, \alpha-\beta+1}\left(A\left(t-t_{j}\right)^{\alpha-\beta}\right) I_{j}\left(x\left(t_{j}^{-}\right)\right) \\
\quad+\int_{0}^{t}(t-s)^{\alpha-1} E_{\alpha-\beta, \alpha}\left(A(t-s)^{\alpha-\beta}\right) B u(s) d s, \\
\quad t \in\left(t_{i}, t_{i+1}\right], \quad i=1,2, \ldots, k .
\end{aligned}\right.
$$

The proof is completed. 
Remark 3.2. The concept of the solution to the homogeneous impulsive problem (3.4) is crucial. Recently, some different views on it, cf. [19], have arisen. However, the authors in [5] have given a clear and correct definition for the concept of the solution of the homogeneous impulsive problem (3.4).

4. Main results. In this section, we present our main results on controllability of linear system (1.1) and nonlinear system (1.2).

First, following [1], we give the concept of controllability as follows.

Definition 4.1. System (1.1) is said to be controllable on $\left[0, t_{f}\right]$, $t_{f} \in(0, T]$, if, for each vector $x_{0}, x_{0}^{\prime}, x_{t_{f}} \in \mathbb{R}^{n}$, there exists a control $u \in L^{1 / p}\left(\left[0, t_{f}\right], \mathbb{R}^{m}\right), p \in(0, \alpha-1)$, such that the corresponding solution of (1.1) satisfies $x\left(t_{f}\right)=x_{t_{f}}$.

4.1. Linear system case. We first give a necessary and sufficient condition of controllability for linear system (1.1).

Theorem 4.2. Linear system (1.1) is controllable on $\left[0, t_{f}\right]$ if and only if the $n \times n$ Gramian matrix

$$
\begin{aligned}
W\left[0, t_{f}\right]=\int_{0}^{t_{f}}\left(t_{f}-s\right)^{2 \alpha-2}\left[E_{\alpha-\beta, \alpha}\right. & \left.\left(A\left(t_{f}-s\right)^{\alpha-\beta}\right) B\right] \\
& \times\left[E_{\alpha-\beta, \alpha}\left(A\left(t_{f}-s\right)^{\alpha-\beta}\right) B\right]^{*} d s
\end{aligned}
$$

is non-singular. Here, $*$ denotes the matrix transpose.

Proof. Sufficiency. Suppose that $W\left[0, t_{f}\right]$ is non-singular. Then its inverse is well defined. For $t_{f} \in\left[0, t_{1}\right]$, define the control function as

$$
u(t)=\left(t_{f}-t\right)^{\alpha-1} B^{*} E_{\alpha-\beta, \alpha}\left(A^{*}\left(t_{f}-t\right)^{\alpha-\beta}\right) W^{-1}\left[0, t_{f}\right] y_{t_{f}},
$$

where

$$
\begin{aligned}
y_{t_{f}}= & x_{t_{f}}-E_{\alpha-\beta}\left(A t_{f}^{\alpha-\beta}\right) x_{0}-A t_{f}^{\alpha-\beta} E_{\alpha-\beta, \alpha-\beta+1}\left(A t_{f}^{\alpha-\beta}\right) x_{0} \\
& +t_{f} E_{\alpha-\beta, 2}\left(A t_{f}^{\alpha-\beta}\right) x_{0}^{\prime} .
\end{aligned}
$$


Substituting $t=t_{f}$ in (3.2) and inserting (4.1) and (4.2), we have

$$
\begin{aligned}
x\left(t_{f}\right)= & E_{\alpha-\beta}\left(A t_{f}^{\alpha-\beta}\right) x_{0}-A t_{f}^{\alpha-\beta} E_{\alpha-\beta, \alpha-\beta+1}\left(A t_{f}^{\alpha-\beta}\right) x_{0} \\
& +t_{f} E_{\alpha-\beta, 2}\left(A t_{f}^{\alpha-\beta}\right) x_{0}^{\prime}+\int_{0}^{t_{f}}\left(t_{f}-s\right)^{\alpha-1} E_{\alpha-\beta, \alpha}\left(A\left(t_{f}-s\right)^{\alpha-\beta}\right) \\
& \times B\left(t_{f}-s\right)^{\alpha-1} B^{*} E_{\alpha-\beta, \alpha}\left(A^{*}\left(t_{f}-s\right)^{\alpha-\beta}\right) W^{-1}\left[0, t_{f}\right] y_{t_{f}} d s \\
= & x_{t_{f}}-y_{t_{f}}+W\left[0, t_{f}\right] W^{-1}\left[0, t_{f}\right] y_{t_{f}}=x_{t_{f}} .
\end{aligned}
$$

Thus, system (1.1) is controllable on $\left[0, t_{f}\right]$ if $t_{f} \in\left[0, t_{1}\right]$.

Next, for $t_{f} \in\left(t_{1}, t_{2}\right]$, define the control function as

$$
\begin{aligned}
& u(t)=\left(t_{f}-t\right)^{\alpha-1} B^{*} E_{\alpha-\beta, \alpha}\left(A^{*}\left(t_{f}-t\right)^{\alpha-\beta}\right) W^{-1}\left[0, t_{f}\right] \\
& {\left[y_{t_{f}}-E_{\alpha-\beta}\left(A\left(t_{f}-t_{1}\right)^{\alpha-\beta}\right) I_{1}\left(x\left(t_{1}^{-}\right)\right)\right.} \\
& \quad+A\left(t_{f}-t_{1}\right)^{\alpha-\beta} E_{\alpha-\beta, \alpha-\beta+1}\left(A\left(t_{f}-t_{1}\right)^{\alpha-\beta}\right) I_{1}\left(x\left(t_{1}^{-}\right)\right) \\
& \left.\quad-\left(t_{f}-t_{1}\right) E_{\alpha-\beta, 2}\left(A\left(t_{f}-t_{1}\right)^{\alpha-\beta}\right) J_{1}\left(x^{\prime}\left(t_{1}^{-}\right)\right)\right] .
\end{aligned}
$$

Substituting $t=t_{f}$ in equation (3.2) and inserting (4.2) and (4.3), we obtain

$$
\begin{aligned}
x\left(t_{f}\right)= & E_{\alpha-\beta}\left(A t_{f}^{\alpha-\beta}\right) x_{0}-A t_{f}^{\alpha-\beta} E_{\alpha-\beta, \alpha-\beta+1}\left(A t_{f}^{\alpha-\beta}\right) x_{0} \\
+ & t_{f} E_{\alpha-\beta, 2}\left(A t_{f}^{\alpha-\beta}\right) x_{0}^{\prime}+E_{\alpha-\beta}\left(A\left(t_{f}-t_{1}\right)^{\alpha-\beta}\right) I_{1}\left(x\left(t_{1}^{-}\right)\right) \\
+ & \left(t_{f}-t_{1}\right) E_{\alpha-\beta, 2}\left(A\left(t_{f}-t_{1}\right)^{\alpha-\beta}\right) J_{1}\left(x^{\prime}\left(t_{1}^{-}\right)\right) \\
& -A\left(t_{f}-t_{1}\right)^{\alpha-\beta} E_{\alpha-\beta, \alpha-\beta+1}\left(A\left(t_{f}-t_{1}\right)^{\alpha-\beta}\right) I_{1}\left(x\left(t_{1}^{-}\right)\right) \\
+ & \int_{0}^{t_{f}}\left(t_{f}-s\right)^{\alpha-1} E_{\alpha-\beta, \alpha}\left(A\left(t_{f}-s\right)^{\alpha-\beta}\right) B\left(t_{f}-s\right)^{\alpha-1} \\
\times & B^{*} E_{\alpha-\beta, \alpha}\left(A^{*}\left(t_{f}-s\right)^{\alpha-\beta}\right) W^{-1}\left[0, t_{f}\right] \\
\times & {\left[y_{t_{f}}+A\left(t_{f}-t_{1}\right)^{\alpha-\beta} E_{\alpha-\beta, \alpha-\beta+1}\left(A\left(t_{f}-t_{1}\right)^{\alpha-\beta}\right) I_{1}\left(x\left(t_{1}^{-}\right)\right)\right.} \\
& -E_{\alpha-\beta}\left(A\left(t_{f}-t_{1}\right)^{\alpha-\beta}\right) I_{1}\left(x\left(t_{1}^{-}\right)\right) \\
& \left.-\left(t_{f}-t_{1}\right) E_{\alpha-\beta, 2}\left(A\left(t_{f}-t_{1}\right)^{\alpha-\beta}\right) J_{1}\left(x^{\prime}\left(t_{1}^{-}\right)\right)\right] d s \\
= & x_{t_{f}}-y_{t_{f}}+E_{\alpha-\beta}\left(A\left(t_{f}-t_{1}\right)^{\alpha-\beta}\right) I_{1}\left(x\left(t_{1}^{-}\right)\right) \\
& -A\left(t_{f}-t_{1}\right)^{\alpha-\beta} E_{\alpha-\beta, \alpha-\beta+1}\left(A\left(t_{f}-t_{1}\right)^{\alpha-\beta}\right) I_{1}\left(x\left(t_{1}^{-}\right)\right) \\
+ & \left(t_{f}-t_{1}\right) E_{\alpha-\beta, 2}\left(A\left(t_{f}-t_{1}\right)^{\alpha-\beta}\right) J_{1}\left(x^{\prime}\left(t_{1}^{-}\right)\right)
\end{aligned}
$$




$$
\begin{aligned}
& +W\left[0, t_{f}\right] W^{-1}\left[0, t_{f}\right]\left[y_{t_{f}}-E_{\alpha-\beta}\left(A\left(t_{f}-t_{1}\right)^{\alpha-\beta}\right) I_{1}\left(x\left(t_{1}^{-}\right)\right)\right. \\
& +A\left(t_{f}-t_{1}\right)^{\alpha-\beta} E_{\alpha-\beta, \alpha-\beta+1}\left(A\left(t_{f}-t_{1}\right)^{\alpha-\beta}\right) I_{1}\left(x\left(t_{1}^{-}\right)\right) \\
& \left.\quad-\left(t_{f}-t_{1}\right) E_{\alpha-\beta, 2}\left(A\left(t_{f}-t_{1}\right)^{\alpha-\beta}\right) J_{1}\left(x^{\prime}\left(t_{1}^{-}\right)\right)\right] \\
& =x_{t_{f}} .
\end{aligned}
$$

Thus system (1.1) is controllable on $\left[0, t_{f}\right]$, if $t_{f} \in\left(t_{1}, t_{2}\right]$.

Moreover, for $t_{f} \in\left(t_{i}, t_{i+1}\right], i=1,2, \ldots, k$, define the control function as

$$
\begin{aligned}
u(t)= & \left(t_{f}-t\right)^{\alpha-1} B^{*} E_{\alpha-\beta, \alpha}\left(A^{*}\left(t_{f}-t\right)^{\alpha-\beta}\right) W^{-1}\left[0, t_{f}\right] \\
& {\left[y_{t_{f}}-\sum_{j=1}^{i} E_{\alpha-\beta}\left(A\left(t_{f}-t_{j}\right)^{\alpha-\beta}\right) I_{j}\left(x\left(t_{j}^{-}\right)\right)\right.} \\
& +A \sum_{j=1}^{i}\left(t_{f}-t_{j}\right)^{\alpha-\beta} E_{\alpha-\beta, \alpha-\beta+1}\left(A\left(t_{f}-t_{j}\right)^{\alpha-\beta}\right) I_{j}\left(x\left(t_{j}^{-}\right)\right) \\
& \left.-\sum_{j=1}^{i}\left(t_{f}-t_{j}\right) E_{\alpha-\beta, 2}\left(A\left(t_{f}-t_{j}\right)^{\alpha-\beta}\right) J_{j}\left(x^{\prime}\left(t_{j}^{-}\right)\right)\right] .
\end{aligned}
$$

Substituting $t=t_{f}$ into (3.2) and inserting (4.2) and (4.4), we have

$$
\begin{aligned}
x\left(t_{f}\right)= & E_{\alpha-\beta}\left(A t_{f}^{\alpha-\beta}\right) x_{0}-A t_{f}^{\alpha-\beta} E_{\alpha-\beta, \alpha-\beta+1}\left(A t_{f}^{\alpha-\beta}\right) x_{0} \\
& +t_{f} E_{\alpha-\beta, 2}\left(A t_{f}^{\alpha-\beta}\right) x_{0}^{\prime}+\sum_{j=1}^{i} E_{\alpha-\beta}\left(A\left(t_{f}-t_{j}\right)^{\alpha-\beta}\right) I_{j}\left(x\left(t_{j}^{-}\right)\right) \\
& +\sum_{j=1}^{i}\left(t_{f}-t_{j}\right) E_{\alpha-\beta, 2}\left(A\left(t_{f}-t_{j}\right)^{\alpha-\beta}\right) J_{j}\left(x^{\prime}\left(t_{j}^{-}\right)\right) \\
& -A \sum_{j=1}^{i}\left(t_{f}-t_{j}\right)^{\alpha-\beta} E_{\alpha-\beta, \alpha-\beta+1}\left(A\left(t_{f}-t_{j}\right)^{\alpha-\beta}\right) I_{j}\left(x\left(t_{j}^{-}\right)\right) \\
& +\int_{0}^{t_{f}}\left(t_{f}-s\right)^{\alpha-1} E_{\alpha-\beta, \alpha}\left(A\left(t_{f}-s\right)^{\alpha-\beta}\right) B\left(t_{f}-s\right)^{\alpha-1} \\
& \times B^{*} E_{\alpha-\beta, \alpha}\left(A^{*}\left(t_{f}-s\right)^{\alpha-\beta}\right) W^{-1}\left[0, t_{f}\right]
\end{aligned}
$$




$$
\begin{aligned}
& \quad\left[y_{t_{f}}-\sum_{j=1}^{i} E_{\alpha-\beta}\left(A\left(t_{f}-t_{j}\right)^{\alpha-\beta}\right) I_{j}\left(x\left(t_{j}^{-}\right)\right)\right. \\
& \quad-\sum_{j=1}^{i}\left(t_{f}-t_{j}\right) E_{\alpha-\beta, 2}\left(A\left(t_{f}-t_{j}\right)^{\alpha-\beta}\right) J_{j}\left(x^{\prime}\left(t_{j}^{-}\right)\right) \\
& \left.\quad+A \sum_{j=1}^{i}\left(t_{f}-t_{j}\right)^{\alpha-\beta} E_{\alpha-\beta, \alpha-\beta+1}\left(A\left(t_{f}-t_{j}\right)^{\alpha-\beta}\right) I_{j}\left(x\left(t_{j}^{-}\right)\right)\right] d s \\
& =x_{t_{f}}-y_{t_{f}}+W\left[0, t_{f}\right] W^{-1}\left[0, t_{f}\right] y_{t_{f}} \\
& =x_{t_{f}} .
\end{aligned}
$$

Thus, system (1.1) is controllable on $\left[0, t_{f}\right]$ if $t_{f} \in(0, T]$.

Necessity. Suppose that system (1.1) is controllable. Now we prove that $W\left[0, t_{f}\right]$ is nonsingular. In fact, if $W\left[0, t_{f}\right]$ is singular, without loss of generality, for $t_{f} \in\left(t_{i}, t_{i+1}\right], i=0,1, \ldots, k$, there exists a nonzero $z_{0}$ such that

$$
z_{0}^{*} W\left[0, t_{f}\right] z_{0}=0
$$

that is,

$$
\begin{aligned}
\int_{0}^{t_{f}} z_{0}^{*}\left(t_{f}-s\right)^{2 \alpha-2} E_{\alpha-\beta, \alpha}(A( & \left.\left.t_{f}-s\right)^{\alpha-\beta}\right) B B^{*} \\
& \times E_{\alpha-\beta, \alpha}\left(A^{*}\left(t_{f}-s\right)^{\alpha-\beta}\right) z_{0} d s=0 .
\end{aligned}
$$

Then, it follows that

$$
z_{0}^{*}\left(t_{f}-s\right)^{\alpha-1} E_{\alpha-\beta, \alpha}\left(A\left(t_{f}-s\right)^{\alpha-\beta}\right) B=0,
$$

on $s \in\left[0, t_{f}\right]$. Since system (1.1) is controllable, then there exists a control $u_{1}(t)$ such that it steers $x_{0}, x_{0}^{\prime} \in \mathbb{R}^{n}$ to the origin $x_{t_{f}}=0$, i.e.,

$$
\begin{aligned}
0= & x\left(t_{f}\right) \\
= & E_{\alpha-\beta}\left(A t_{f}^{\alpha-\beta}\right) x_{0}+t_{f} E_{\alpha-\beta, 2}\left(A t_{f}^{\alpha-\beta}\right) x_{0}^{\prime} \\
& -A t_{f}^{\alpha-\beta} E_{\alpha-\beta, \alpha-\beta+1}\left(A t_{f}^{\alpha-\beta}\right) x_{0} \\
& +\sum_{j=1}^{i} E_{\alpha-\beta}\left(A\left(t_{f}-t_{j}\right)^{\alpha-\beta}\right) I_{j}\left(x\left(t_{j}^{-}\right)\right)
\end{aligned}
$$




$$
\begin{aligned}
& +\sum_{j=1}^{i}\left(t_{f}-t_{j}\right) E_{\alpha-\beta, 2}\left(A\left(t_{f}-t_{j}\right)^{\alpha-\beta}\right) J_{j}\left(x^{\prime}\left(t_{j}^{-}\right)\right) \\
& -A \sum_{j=1}^{i}\left(t_{f}-t_{j}\right)^{\alpha-\beta} E_{\alpha-\beta, \alpha-\beta+1}\left(A\left(t_{f}-t_{j}\right)^{\alpha-\beta}\right) I_{j}\left(x\left(t_{j}^{-}\right)\right) \\
& +\int_{0}^{t_{f}}\left(t_{f}-s\right)^{\alpha-1} E_{\alpha-\beta, \alpha}\left(A\left(t_{f}-s\right)^{\alpha-\beta}\right) B u_{1}(s) d s .
\end{aligned}
$$

Similarly, for the above vectors $x_{0}, x_{0}^{\prime} \in \mathbb{R}^{n}$ and the final state $x_{t_{f}}^{*}=z_{0}$, there is also a control $u_{2}(t)$ such that

$$
\begin{aligned}
z_{0}= & x\left(t_{f}\right) \\
= & E_{\alpha-\beta}\left(A t_{f}^{\alpha-\beta}\right) x_{0}+t_{f} E_{\alpha-\beta, 2}\left(A t_{f}^{\alpha-\beta}\right) x_{0}^{\prime} \\
& -A t_{f}^{\alpha-\beta} E_{\alpha-\beta, \alpha-\beta+1}\left(A t_{f}^{\alpha-\beta}\right) x_{0} \\
& +\sum_{j=1}^{i} E_{\alpha-\beta}\left(A\left(t_{f}-t_{j}\right)^{\alpha-\beta}\right) I_{j}\left(x\left(t_{j}^{-}\right)\right) \\
& +\sum_{j=1}^{i}\left(t_{f}-t_{j}\right) E_{\alpha-\beta, 2}\left(A\left(t_{f}-t_{j}\right)^{\alpha-\beta}\right) J_{j}\left(x^{\prime}\left(t_{j}^{-}\right)\right) \\
& -A \sum_{j=1}^{i}\left(t_{f}-t_{j}\right)^{\alpha-\beta} E_{\alpha-\beta, \alpha-\beta+1}\left(A\left(t_{f}-t_{j}\right)^{\alpha-\beta}\right) I_{j}\left(x\left(t_{j}^{-}\right)\right) \\
& +\int_{0}^{t_{f}}\left(t_{f}-s\right)^{\alpha-1} E_{\alpha-\beta, \alpha}\left(A\left(t_{f}-s\right)^{\alpha-\beta}\right) B u_{2}(s) d s .
\end{aligned}
$$

We obtain the following from (4.5) and (4.6).

$$
z_{0}+\int_{0}^{t_{f}}\left(t_{f}-s\right)^{\alpha-1} E_{\alpha-\beta, \alpha}\left(A\left(t_{f}-s\right)^{\alpha-\beta}\right) B\left(u_{1}(s)-u_{2}(s)\right) d s=0 .
$$

Multiplying $z_{0}^{*}$ on both sides of (4.7), we obtain

$$
z_{0}^{*} z_{0}+\int_{0}^{t_{f}}\left(t_{f}-s\right)^{\alpha-1} z_{0}^{*} E_{\alpha-\beta, \alpha}\left(A\left(t_{f}-s\right)^{\alpha-\beta}\right) B\left(u_{1}(s)-u_{2}(s)\right) d s=0 .
$$

By the fact that $z_{0}^{*} W\left[0, t_{f}\right] z_{0}=0$, we have $z_{0}^{*} z_{0}=0$. Thus, $z_{0}=0$. This is a contraction. The proof is completed. 
Theorem 4.2 is a geometric type condition. By simple transformation, we can get an algebraic type condition.

Theorem 4.3. System (1.1) is controllable on $\left[0, t_{f}\right], t_{f} \in(0, T]$, if and only if

$$
\operatorname{rank} Q=\operatorname{rank}\left(B|A B| \cdots \mid A^{n-1} B\right)=n ;
$$

here $\left(B|A B| \cdots \mid A^{n-1} B\right)$ is an $n \times n m$ matrix composed by the column vectors of $B, \ldots, A^{n-1} B$ and the mark $\mid$ denotes the block of the matrix.

Proof. By the Cayley-Hamilton theorem, we have

$$
t^{\alpha-1} E_{\alpha-\beta, \alpha}\left(A t^{\alpha-\beta}\right)=\sum_{m=0}^{\infty} \frac{t^{(\alpha-\beta) m+\alpha-1}}{\Gamma(m(\alpha-\beta)+\alpha)} A^{m}=\sum_{m=0}^{n-1} c_{m}(t) A^{m},
$$

where $c_{m}(t)$ is a polynomial in $t$, and it follows that

$$
\left(t_{f}-s\right)^{\alpha-1} E_{\alpha-\beta, \alpha}\left(A\left(t_{f}-s\right)^{\alpha-\beta}\right)=\sum_{m=0}^{n-1} c_{m}\left(t_{f}-s\right) A^{m} .
$$

By Theorem 4.2, for $t_{f} \in\left[0, t_{1}\right]$, we have

$$
\begin{aligned}
x_{t_{f}}= & E_{\alpha-\beta}\left(A t_{f}^{\alpha-\beta}\right) x_{0} \\
& +t_{f} E_{\alpha-\beta, 2}\left(A t_{f}^{\alpha-\beta}\right) x_{0}^{\prime}-A t_{f}^{\alpha-\beta} E_{\alpha-\beta, \alpha-\beta+1}\left(A t_{f}^{\alpha-\beta}\right) x_{0} \\
& +\int_{0}^{t_{f}}\left(t_{f}-s\right)^{\alpha-1} E_{\alpha-\beta, \alpha}\left(A\left(t_{f}-s\right)^{\alpha-\beta}\right) B u(s) d s .
\end{aligned}
$$

For convenience, we denote

$$
\begin{aligned}
\eta_{1}=E_{\alpha-\beta}\left(A t_{f}^{\alpha-\beta}\right) x_{0}+t_{f} E_{\alpha-\beta, 2}\left(A t_{f}^{\alpha-\beta}\right) x_{0}^{\prime} & \\
& -A t_{f}^{\alpha-\beta} E_{\alpha-\beta, \alpha-\beta+1}\left(A t_{f}^{\alpha-\beta}\right) x_{0} .
\end{aligned}
$$

Combining (4.8) and (4.9) yields

$$
\begin{aligned}
x_{t_{f}}-\eta_{1} & =\int_{0}^{t_{f}}\left(t_{f}-s\right)^{\alpha-1} E_{\alpha-\beta, \alpha}\left(A\left(t_{f}-s\right)^{\alpha-\beta}\right) B u(s) d s \\
& =\sum_{m=0}^{n-1} A^{m} B \int_{0}^{t_{f}} c_{m}\left(t_{f}-s\right) u(s) d s
\end{aligned}
$$




$$
=\left(B|A B| \cdots \mid A^{n-1} B\right)\left(\begin{array}{c}
d_{0} \\
d_{1} \\
\vdots \\
d_{n-1}
\end{array}\right) \text {, }
$$

where

$$
d_{m}=\int_{0}^{t_{f}} c_{m}\left(t_{f}-s\right) u(s) d s .
$$

Note that $x_{t_{f}}, x_{0}$ and $x_{0}^{\prime}$ are arbitrary; to have a unique solution of $u(t)$, the necessary and sufficient condition is clearly that

$$
\operatorname{rank} Q=\operatorname{rank}\left(B|A B| \cdots \mid A^{n-1} B\right)=n .
$$

For $t_{f} \in\left(t_{i}, t_{i+1}\right], i=1,2, \ldots, k$, we obtain

$$
\begin{aligned}
x_{t_{f}}= & E_{\alpha-\beta}\left(A t_{f}^{\alpha-\beta}\right) x_{0}+t_{f} E_{\alpha-\beta, 2}\left(A t_{f}^{\alpha-\beta}\right) x_{0}^{\prime} \\
& -A t_{f}^{\alpha-\beta} E_{\alpha-\beta, \alpha-\beta+1}\left(A t_{f}^{\alpha-\beta}\right) x_{0} \\
& +\sum_{j=1}^{i} E_{\alpha-\beta}\left(A\left(t_{f}-t_{j}\right)^{\alpha-\beta}\right) I_{j}\left(x\left(t_{j}^{-}\right)\right) \\
& +\sum_{j=1}^{i}\left(t_{f}-t_{j}\right) E_{\alpha-\beta, 2}\left(A\left(t_{f}-t_{j}\right)^{\alpha-\beta}\right) J_{j}\left(x^{\prime}\left(t_{j}^{-}\right)\right) \\
& -A \sum_{j=1}^{i}\left(t_{f}-t_{j}\right)^{\alpha-\beta} E_{\alpha-\beta, \alpha-\beta+1}\left(A\left(t_{f}-t_{j}\right)^{\alpha-\beta}\right) I_{j}\left(x\left(t_{j}^{-}\right)\right) \\
& +\int_{0}^{t_{f}}\left(t_{f}-s\right)^{\alpha-1} E_{\alpha-\beta, \alpha}\left(A\left(t_{f}-s\right)^{\alpha-\beta}\right) B u(s) d s .
\end{aligned}
$$

Denote

$$
\begin{aligned}
\eta_{i}= & E_{\alpha-\beta}\left(A t_{f}^{\alpha-\beta}\right) x_{0}+t_{f} E_{\alpha-\beta, 2}\left(A t_{f}^{\alpha-\beta}\right) x_{0}^{\prime} \\
& -A t_{f}^{\alpha-\beta} E_{\alpha-\beta, \alpha-\beta+1}\left(A t_{f}^{\alpha-\beta}\right) x_{0} \\
+ & \sum_{j=1}^{i} E_{\alpha-\beta}\left(A\left(t_{f}-t_{j}\right)^{\alpha-\beta}\right) I_{j}\left(x\left(t_{j}^{-}\right)\right)
\end{aligned}
$$




$$
\begin{aligned}
& +\sum_{j=1}^{i}\left(t_{f}-t_{j}\right) E_{\alpha-\beta, 2}\left(A\left(t_{f}-t_{j}\right)^{\alpha-\beta}\right) J_{j}\left(x^{\prime}\left(t_{j}^{-}\right)\right) \\
& -A \sum_{j=1}^{i}\left(t_{f}-t_{j}\right)^{\alpha-\beta} E_{\alpha-\beta, \alpha-\beta+1}\left(A\left(t_{f}-t_{j}\right)^{\alpha-\beta}\right) I_{j}\left(x\left(t_{j}^{-}\right)\right) .
\end{aligned}
$$

Combining equations (4.10) and (4.11), we have

$$
\begin{aligned}
x_{t_{f}}-\eta_{i} & =\int_{0}^{t_{f}}\left(t_{f}-s\right)^{\alpha-1} E_{\alpha-\beta, \alpha}\left(A\left(t_{f}-s\right)^{\alpha-\beta}\right) B u(s) d s \\
& =\sum_{m=0}^{n-1} A^{m} B \int_{0}^{t_{f}} c_{m}\left(t_{f}-s\right) u(s) d s \\
& =\left(B|A B| \cdots \mid A^{n-1} B\right)\left(\begin{array}{c}
d_{0} \\
d_{1} \\
\vdots \\
d_{n-1}
\end{array}\right) .
\end{aligned}
$$

Note that $x_{t_{f}}, x_{0}$ and $x_{0}^{\prime}$ are arbitrary. In order to have a unique solution of $u(t)$, the necessary and sufficient condition is clear that

$$
\operatorname{rank} Q=\operatorname{rank}\left(B|A B| \cdots \mid A^{n-1} B\right)=n .
$$

The proof is completed.

4.2. Nonlinear system case. Here, we study the controllability of the nonlinear system (1.2) by Schauder's fixed point theorem. In order to obtain the main result we make the following assumptions:

$H(1)$ : The function $f: J \times \mathbb{R}^{n} \rightarrow \mathbb{R}^{n}$ satisfies the following:

(i) $f$ is measurable for all $t \in J$ and for every fixed $x \in \mathbb{R}^{n}$.

(ii) There exists a constant $L_{f}>0$ such that, for almost every $t \in J$,

$$
\|f(t, x)-f(t, y)\| \leq L_{f}\|x-y\|, \quad \text { for all } x, y \in \mathbb{R}^{n} .
$$

(iii) There exist a function $\Psi \in L^{1 / \gamma}\left(J, \mathbb{R}_{+}\right), \gamma \in(0, \alpha-1)$ and a constant $\theta>0$, such that

$$
\|f(t, x)\| \leq \Psi(t)+\theta\|x\|, \quad \text { for almost every } t \in J .
$$

$H(2): I_{i}, J_{i}: \mathbb{R}^{n} \rightarrow \mathbb{R}^{n}, i=1,2, \ldots, k$, satisfy the following: $I_{i}$ and $J_{i}$ are continuous, and there exist constants $h_{i}, d_{i}>0, i=1, \ldots, k$, 
such that

$$
\begin{aligned}
\left\|I_{i}(x)-I_{i}(y)\right\| \leq h_{i}\|x-y\|, & \text { for all } x, y \in \mathbb{R}^{n}, \\
\left\|J_{i}(x)-J_{i}(y)\right\| \leq d_{i}\|x-y\|, & \text { for all } x, y \in \mathbb{R}^{n} .
\end{aligned}
$$

In the sequel, for convenience, let us set

$$
\begin{aligned}
& \|I(0)\|=\sup \left\{\left\|I_{i}(0)\right\|\right\}, \quad\|J(0)\|=\sup \left\{\left\|J_{i}(0)\right\|\right\} \quad i=1,2, \ldots, k ; \\
& a_{1}=\sup _{t \in J}\left\|E_{\alpha-\beta}\left(A t^{\alpha-\beta}\right)\right\|, \\
& a_{2}=\sup _{t \in J}\left\|A E_{\alpha-\beta, \alpha-\beta+1}\left(A t^{\alpha-\beta}\right) t^{\alpha-\beta}\right\| ; \\
& a_{3}=\sup _{t \in J}\left\|E_{\alpha-\beta, 2}\left(A t^{\alpha-\beta}\right) t\right\|, \\
& a_{4}=\sup _{t \in J}\left\|(T-t)^{\alpha-1} E_{\alpha-\beta, \alpha}\left(A(T-t)^{\alpha-\beta}\right)\right\| ; \\
& a_{5}=\sup _{t \in J}\left\|E_{\alpha-\beta, \alpha-1}\left(A(T-t)^{\alpha-\beta}\right)\right\|, \\
& b=\max \left\{a_{1}, a_{2}, a_{3}, a_{4}, a_{5}\right\} ; \\
& a_{6}=\sup _{t \in J}\left\|(T-t)^{\alpha-1} B^{*} E_{\alpha-\beta, \alpha}\left(A^{*}(T-t)^{\alpha-\beta}\right) W^{-1}[0, T]\right\| ; \\
& a_{7}=\left\|y_{T}\right\| \text {, } \\
& \delta_{1}=\left(\frac{1-\gamma}{\alpha-1-\gamma} T^{(\alpha-1-\gamma) /(1-\gamma)}\right)^{1-\gamma}, \quad \delta_{2}=\max \left\{T, \frac{T^{\alpha-1}}{\alpha-1}\right\} \\
& \|B\|=M_{B} \\
& c_{1}=1+b M_{B} T a_{6}, \quad c_{2}=1+b M_{B} \frac{T^{\alpha-1}}{\alpha-1} a_{6}, \\
& c=\max \left\{c_{1}, c_{2}\right\} \\
& \rho_{1}=T^{1-\gamma}+b M_{B} T^{2-\gamma} a_{6}, \quad \rho_{2}=\delta_{1}+b M_{B} \frac{T^{\alpha-\gamma}}{\alpha-1} a_{6}, \\
& \rho=\max \left\{\rho_{1}, \rho_{2}\right\} \text {. }
\end{aligned}
$$

Now, we are in a position to provide the main result of this part.

Theorem 4.4. If $H(1)$ and $H(2)$ are satisfied and the linear system (1.1) is controllable on $\left[0, t_{f}\right]$ for some $t_{f} \in(0, T]$, then system $(1.2)$ is 
also controllable on $\left[0, t_{f}\right]$, provided that

$$
\left(\sum_{i=1}^{k}\left(2 h_{i}+d_{i}\right)+\theta T\right) b c<1 .
$$

Proof. From the controllability of linear system (1.1) on $\left[0, t_{f}\right]$ for $t_{f} \in\left(t_{i}, t_{i+1}\right] \subset(0, T]$, we can define the control as follows:

$$
u(t)=\left\{\begin{array}{l}
(T-t)^{\alpha-1} B^{*} E_{\alpha-\beta, \alpha}\left(A^{*}(T-t)^{\alpha-\beta}\right) W^{-1}[0, T] \\
\times\left[y_{t_{f}}-\int_{0}^{T}(T-s)^{\alpha-1} E_{\alpha-\beta, \alpha}\left(A(T-s)^{\alpha-\beta}\right) f(s, x(s)) d s\right], \\
\quad t \in\left[0, t_{1}\right], \\
(T-t)^{\alpha-1} B^{*} E_{\alpha-\beta, \alpha}\left(A^{*}(T-t)^{\alpha-\beta}\right) W^{-1}[0, T] \\
\times\left[y_{t_{f}}-\sum_{j=1}^{i} E_{\alpha-\beta}\left(A\left(T-t_{j}\right)^{\alpha-\beta}\right) I_{j}\left(x\left(t_{j}^{-}\right)\right)\right. \\
-\sum_{j=1}^{i}\left(T-t_{j}\right) E_{\alpha-\beta, 2}\left(A\left(T-t_{j}\right)^{\alpha-\beta}\right) J_{j}\left(x^{\prime}\left(t_{j}^{-}\right)\right) \\
+A \sum_{j=1}^{i}\left(T-t_{j}\right)^{\alpha-\beta} E_{\alpha-\beta, \alpha-\beta+1}\left(A\left(T-t_{j}\right)^{\alpha-\beta}\right) I_{j}\left(x\left(t_{j}^{-}\right)\right) \\
\left.-\int_{0}^{T}(T-s)^{\alpha-1} E_{\alpha-\beta, \alpha}\left(A(T-s)^{\alpha-\beta}\right) f(s, x(s)) d s\right], \\
\quad t \in\left(t_{i}, t_{i+1}\right], \quad i=1,2, \ldots, k,
\end{array}\right.
$$

where

$$
\begin{aligned}
y_{t_{f}}= & x_{t_{f}}-E_{\alpha-\beta}\left(A T^{\alpha-\beta}\right) x_{0}-A T^{\alpha-\beta} E_{\alpha-\beta, \alpha-\beta+1}\left(A T^{\alpha-\beta}\right) x_{0} \\
& +T E_{\alpha-\beta, 2}\left(A T^{\alpha-\beta}\right) x_{0}^{\prime} .
\end{aligned}
$$

According to this control, the operator $F: P C^{1}\left(J, \mathbb{R}^{n}\right) \rightarrow P C^{1}\left(J, \mathbb{R}^{n}\right)$ is well defined by 


$$
(F x)(t)=\left\{\begin{aligned}
E_{\alpha-\beta}\left(A t^{\alpha-\beta}\right) x_{0}-A t^{\alpha-\beta} E_{\alpha-\beta, \alpha-\beta+1}\left(A t^{\alpha-\beta}\right) x_{0} \\
\quad+t E_{\alpha-\beta, 2}\left(A t^{\alpha-\beta}\right) x_{0}^{\prime} \\
+\int_{0}^{t}(t-s)^{\alpha-1} E_{\alpha-\beta, \alpha}\left(A(t-s)^{\alpha-\beta}\right)[B u(s)+f(s, x(s))] d s \\
\quad t \in\left[0, t_{1}\right], \\
E_{\alpha-\beta}\left(A t^{\alpha-\beta}\right) x_{0}-A t^{\alpha-\beta} E_{\alpha-\beta, \alpha-\beta+1}\left(A t^{\alpha-\beta}\right) x_{0} \\
+t E_{\alpha-\beta, 2}\left(A t^{\alpha-\beta}\right) x_{0}^{\prime}+\sum_{j=1}^{i} E_{\alpha-\beta}\left(A\left(t-t_{j}\right)^{\alpha-\beta}\right) I_{j}\left(x\left(t_{j}^{-}\right)\right) \\
+\sum_{j=1}^{i}\left(t-t_{j}\right) E_{\alpha-\beta, 2}\left(A\left(t-t_{j}\right)^{\alpha-\beta}\right) J_{j}\left(x^{\prime}\left(t_{j}^{-}\right)\right) \\
\quad-A \sum_{j=1}^{i}\left(t-t_{j}\right)^{\alpha-\beta} E_{\alpha-\beta, \alpha-\beta+1}\left(A\left(t-t_{j}\right)^{\alpha-\beta}\right) I_{j}\left(x\left(t_{j}^{-}\right)\right) \\
+\int_{0}^{t}(t-s)^{\alpha-1} E_{\alpha-\beta, \alpha}\left(A(t-s)^{\alpha-\beta}\right)[B u(s)+f(s, x(s))] d s, \\
\quad t \in\left(t_{i}, t_{i+1}\right], \quad i=1,2, \ldots, k .
\end{aligned}\right.
$$

We easily observe that $(F x)\left(t_{f}\right)=x_{t_{f}}$. Hence, in order to prove the controllability of system (1.2), we only need to show that $F$ has a fixed point.

For $t_{f} \in\left(0, t_{1}\right]$, we know that system $(1.2)$ is controllable on $\left[0, t_{f}\right]$ from [2]. Now we show system (1.2) is controllable on $\left[0, t_{f}\right]$ for $t_{f} \in\left(t_{i}, t_{i+1}\right], i=1, \ldots, k$.

First, we choose

$$
\begin{array}{r}
b\left[\left(2\left\|x_{0}\right\|+\left\|x_{0}^{\prime}\right\|\right)+k c(2\|I(0)\|+\|J(0)\|)+\rho\|\Psi\|_{L^{1 / \gamma}}+M_{B} \delta_{2} a_{6} a_{7}\right] \\
\times\left(1-b c \sum_{i=1}^{k}\left(2 h_{i}+d_{i}\right)-b c T \theta\right)^{-1} \leq r,
\end{array}
$$

and consider the bounded set $B_{r}=\left\{x \in P C^{1}\left(J, \mathbb{R}^{n}\right):\|x\|_{P C^{1}} \leq r\right\}$. Next, we divide the proof into three steps.

Step 1: We prove that $F B_{r} \subseteq B_{r}$. For all $x \in B_{r}$ and for all $t \in\left(t_{i}, t_{i+1}\right], i=1,2, \ldots, k$, we have

$$
\begin{aligned}
\|u(t)\| \leq & \left\|(T-t)^{\alpha-1} B^{*} E_{\alpha-\beta, \alpha}\left(A^{*}(T-t)^{\alpha-\beta}\right) W^{-1}[0, T]\right\| \\
\times & {\left[\left\|y_{t_{f}}\right\|+\left\|\sum_{j=1}^{i} E_{\alpha-\beta}\left(A\left(T-t_{j}\right)^{\alpha-\beta}\right) I_{j}\left(x\left(t_{j}^{-}\right)\right)\right\|\right.} \\
& +\left\|\sum_{j=1}^{i}\left(T-t_{j}\right) E_{\alpha-\beta, 2}\left(A\left(T-t_{j}\right)^{\alpha-\beta}\right) J_{j}\left(x^{\prime}\left(t_{j}^{-}\right)\right)\right\|
\end{aligned}
$$




$$
\begin{aligned}
& +\left\|A \sum_{j=1}^{i}\left(T-t_{j}\right)^{\alpha-\beta} E_{\alpha-\beta, \alpha-\beta+1}\left(A\left(T-t_{j}\right)^{\alpha-\beta}\right) I_{j}\left(x\left(t_{j}^{-}\right)\right)\right\| \\
& \left.+\left\|\int_{0}^{T}(T-s)^{\alpha-1} E_{\alpha-\beta, \alpha}\left(A(T-s)^{\alpha-\beta}\right) f(s, x(s)) d s\right\|\right] \\
& \leq a_{6} \times\left[a_{7}+b \sum_{i=1}^{k} h_{i}\left\|x\left(t_{i}^{-}\right)\right\|+b k\|I(0)\|\right. \\
& +b \sum_{i=1}^{k} d_{i}\left\|x^{\prime}\left(t_{i}^{-}\right)\right\|+b k\|J(0)\|+b \sum_{i=1}^{k} h_{i}\left\|x\left(t_{i}^{-}\right)\right\|+b k\|I(0)\| \\
& \left.+b \int_{0}^{T}[\Psi(s)+\theta\|x(s)\|] d s\right] \\
& \leq a_{6} \times\left[a_{7}+b k(2\|I(0)\|+\|J(0)\|)+b \sum_{i=1}^{k}\left(2 h_{i}+d_{i}\right) r\right. \\
& \left.+b T^{1-\gamma}\|\Psi\|_{L^{1 / \gamma}}+b T \theta r\right] .
\end{aligned}
$$

From the above estimate, we have

$$
\begin{aligned}
\|(F x)(t)\| \leq & \left\|E_{\alpha-\beta}\left(A t^{\alpha-\beta}\right) x_{0}\right\| \\
& +\left\|t E_{\alpha-\beta, 2}\left(A t^{\alpha-\beta}\right) x_{0}^{\prime}\right\| \\
& +\left\|A t^{\alpha-\beta} E_{\alpha-\beta, \alpha-\beta+1}\left(A t^{\alpha-\beta}\right) x_{0}\right\| \\
& +\left\|\sum_{j=1}^{i} E_{\alpha-\beta}\left(A\left(t-t_{j}\right)^{\alpha-\beta}\right) I_{j}\left(x\left(t_{j}^{-}\right)\right)\right\| \\
& +\left\|\sum_{j=1}^{i}\left(t-t_{j}\right) E_{\alpha-\beta, 2}\left(A\left(t-t_{j}\right)^{\alpha-\beta}\right) J_{j}\left(x^{\prime}\left(t_{j}^{-}\right)\right)\right\| \\
& +\left\|A \sum_{j=1}^{i}\left(t-t_{j}\right)^{\alpha-\beta} E_{\alpha-\beta, \alpha-\beta+1}\left(A\left(t-t_{j}\right)^{\alpha-\beta}\right) I_{j}\left(x\left(t_{j}^{-}\right)\right)\right\| \\
& +\left\|\int_{0}^{t}(t-s)^{\alpha-1} E_{\alpha-\beta, \alpha}\left(A(t-s)^{\alpha-\beta}\right)[B u(s)+f(s, x(s))] d s\right\| \\
\leq & b\left(2\left\|x_{0}\right\|+\left\|x_{0}^{\prime}\right\|\right)+b \sum_{i=1}^{k} h_{i}\left\|x\left(t_{i}^{-}\right)\right\|+b k\|I(0)\|
\end{aligned}
$$




$$
\begin{aligned}
& +b \sum_{i=1}^{k} d_{i}\left\|x^{\prime}\left(t_{i}^{-}\right)\right\|+b k\|J(0)\|+b \sum_{i=1}^{k} h_{i}\left\|x\left(t_{i}^{-}\right)\right\|+b k\|I(0)\| \\
& +b\left\|\int_{0}^{t} B u(s) d s\right\|+b \int_{0}^{t}[\Psi(s)+\theta\|x(s)\|] d s \\
& \leq b\left(2\left\|x_{0}\right\|+\left\|x_{0}^{\prime}\right\|\right)+b k(2\|I(0)\|+\|J(0)\|)+b \sum_{i=1}^{k}\left(2 h_{i}+d_{i}\right)\|x\| \\
& +b M_{B} T a_{6} \times\left[a_{7}+b k(2\|I(0)\|+\|J(0)\|)\right. \\
& \left.+b \sum_{i=1}^{k}\left(2 h_{i}+d_{i}\right) r+b T^{1-\gamma}\|\Psi\|_{L^{1 / \gamma}}+b T \theta r\right] \\
& +b T^{1-\gamma}\|\Psi\|_{L^{1 / \gamma}}+b \int_{0}^{t} \theta\|x(s)\| d s \\
& \leq b\left(2\left\|x_{0}\right\|+\left\|x_{0}^{\prime}\right\|\right)+b k\left(1+b M_{B} T a_{6}\right)(2\|I(0)\|+\|J(0)\|) \\
& +b M_{B} T a_{6} a_{7}+b\left(T^{1-\gamma}+b M_{B} T^{2-\gamma} a_{6}\right)\|\Psi\|_{L^{1 / \gamma}} \\
& +b\left(1+b M_{B} T a_{6}\right)\left(\sum_{i=1}^{k}\left(2 h_{i}+d_{i}\right)+T \theta\right) r \\
& \leq b\left(2\left\|x_{0}\right\|+\left\|x_{0}^{\prime}\right\|\right)+b k c(2\|I(0)\|+\|J(0)\|)+b \rho\|\Psi\|_{L^{1 / \gamma}} \\
& +b M_{B} \delta_{2} a_{6} a_{7}+b c\left(\sum_{i=1}^{k}\left(2 h_{i}+d_{i}\right)+T \theta\right) r \leq r .
\end{aligned}
$$

Next, from the definition of $F$ and the Mittag-Leffer function, we obtain:

$$
\begin{aligned}
\left\|(F x)^{\prime}(t)\right\| \leq & \left\|E_{\alpha-\beta}\left(A t^{\alpha-\beta}\right) x_{0}^{\prime}\right\| \\
& +\left\|\sum_{j=1}^{i} E_{\alpha-\beta}\left(A\left(t-t_{j}\right)^{\alpha-\beta}\right) J_{j}\left(x^{\prime}\left(t_{j}^{-}\right)\right)\right\| \\
& +\left\|\int_{0}^{t}(t-s)^{\alpha-2} E_{\alpha-\beta, \alpha-1}\left(A(t-s)^{\alpha-\beta}\right)[B u(s)+f(s, x(s))] d s\right\| \\
\leq & b\left\|x_{0}^{\prime}\right\|+b \sum_{i=1}^{k} d_{i}\left\|x^{\prime}\left(t_{i}^{-}\right)\right\|+b k\|J(0)\|
\end{aligned}
$$




$$
\begin{aligned}
& +b M_{B}\left\|\int_{0}^{t}(t-s)^{\alpha-2} u(s) d s\right\| \\
& +b \int_{0}^{t}(t-s)^{\alpha-2}[\Psi(s)+\theta\|x(s)\|] d s \\
& \leq b\left\|x_{0}^{\prime}\right\|+b \sum_{i=1}^{k} d_{i}\left\|x^{\prime}\right\|+b k\|J(0)\|+\frac{b M_{B}\|u\| T^{\alpha-1}}{\alpha-1} \\
& +b\|\Psi\|_{L^{1 / \gamma}}\left(\frac{1-\gamma}{\alpha-1-\gamma} T^{(\alpha-1-\gamma) /(1-\gamma)}\right)^{1-\gamma}+b T \theta\|x\| \\
& \leq b\left\|x_{0}^{\prime}\right\|+b k\|J(0)\|+\frac{b M_{B} T^{\alpha-1} a_{6}}{\alpha-1} \\
& {\left[a_{7}+b k(2\|I(0)\|+\|J(0)\|)\right.} \\
& \left.+b \sum_{i=1}^{k}\left(2 h_{i}+d_{i}\right) r+b T^{1-\gamma}\|\Psi\|_{L^{1 / \gamma}}+b T \theta r\right] \\
& +b\|\Psi\|_{L^{1 / \gamma}} \delta_{1}+b\left(\sum_{i=1}^{k} d_{i}+\theta T\right) r \\
& \leq b\left\|x_{0}^{\prime}\right\|+k b\left(1+\frac{b M_{B} T^{\alpha-1} a_{6}}{\alpha-1}\right)(2\|I(0)\|+\|J(0)\|) \\
& +b\left(\delta_{1}+\frac{b M_{B} T^{\alpha-\gamma} a_{6}}{\alpha-1}\right)\|\Psi\|_{L^{1 / \gamma}}+\frac{b M_{B} T^{\alpha-1} a_{6} a_{7}}{\alpha-1} \\
& +b\left(1+\frac{b M_{B} T^{\alpha-1} a_{6}}{\alpha-1}\right)\left(\sum_{i=1}^{k}\left(2 h_{i}+d_{i}\right)+\theta T\right) r \\
& \leq b\left\|x_{0}^{\prime}\right\|+k b c(2\|I(0)\|+\|J(0)\|)+b \rho\|\Psi\|_{L^{1 / \gamma}}+b M_{B} \delta_{2} a_{6} a_{7} \\
& +b c\left(\sum_{i=1}^{k}\left(2 h_{i}+d_{i}\right)+\theta T\right) r \\
& \leq r .
\end{aligned}
$$

Hence, according to the above, we obtain $\|F x\|_{P C^{1}} \leq r$, which means that $F B_{r} \subseteq B_{r}$.

Step 2: We show that $F$ is continuous. Let $\left\{x_{n}\right\}$ be a sequence such that $x_{n} \rightarrow x, x_{n}^{\prime} \rightarrow x^{\prime}$ in $P C^{1}\left(J, \mathbb{R}^{n}\right)$ as $n \rightarrow \infty$. Then, for each $t \in\left(t_{i}, t_{i+1}\right], i=1,2, \ldots, k$, we obtain: 


$$
\begin{aligned}
& \left\|\left(F x_{n}\right)(t)-(F x)(t)\right\| \\
& \leq\left\|\sum_{j=1}^{i} E_{\alpha-\beta}\left(A\left(t-t_{j}\right)^{\alpha-\beta}\right)\left[I_{j}\left(x_{n}\left(t_{j}^{-}\right)\right)-I_{j}\left(x\left(t_{j}^{-}\right)\right)\right]\right\| \\
& +\left\|\sum_{j=1}^{i}\left(t-t_{j}\right) E_{\alpha-\beta, 2}\left(A\left(t-t_{j}\right)^{\alpha-\beta}\right)\left[J_{j}\left(x_{n}^{\prime}\left(t_{j}^{-}\right)\right)-J_{j}\left(x^{\prime}\left(t_{j}^{-}\right)\right)\right]\right\| \\
& +\| A \sum_{j=1}^{i}\left(t-t_{j}\right)^{\alpha-\beta} E_{\alpha-\beta, \alpha-\beta+1}\left(A\left(t-t_{j}\right)^{\alpha-\beta}\right) \\
& {\left[I_{j}\left(x_{n}\left(t_{j}^{-}\right)\right)-I_{j}\left(x\left(t_{j}^{-}\right)\right)\right] \|} \\
& \left.+\| \int_{0}^{t}(t-s)^{\alpha-1} E_{\alpha-\beta, \alpha}\left(A(t-s)^{\alpha-\beta}\right)\left[f\left(s, x_{n}(s)\right)-f(s, x(s))\right]\right) d s \| \\
& \leq b \sum_{i=1}^{k} h_{i}\left\|x_{n}\left(t_{i}^{-}\right)-x\left(t_{i}^{-}\right)\right\|+b \sum_{i=1}^{k} d_{i}\left\|x_{n}^{\prime}\left(t_{i}^{-}\right)-x^{\prime}\left(t_{i}^{-}\right)\right\| \\
& +b \sum_{i=1}^{k} h_{i}\left\|x_{n}\left(t_{i}^{-}\right)-x\left(t_{i}^{-}\right)\right\|+b L_{f} \int_{0}^{t}\left\|x_{n}(s)-x(s)\right\| d s \\
& \leq b\left(\sum_{i=1}^{k} 2 h_{i}+L_{f} T\right)\left\|x_{n}-x\right\|+b \sum_{i=1}^{k} d_{i}\left\|x_{n}^{\prime}-x^{\prime}\right\|, \\
& \left\|\left(F x_{n}\right)^{\prime}(t)-(F x)^{\prime}(t)\right\| \\
& \leq \| \sum_{j=1}^{i}\left(t-t_{j}\right) E_{\alpha-\beta}\left(A\left(t-t_{j}^{\alpha-\beta}\right)\left[J_{j}\left(x_{n}^{\prime}\left(t_{j}^{-}\right)\right)-J_{j}\left(x^{\prime}\left(t_{j}^{-}\right)\right)\right] \|\right. \\
& +\left\|\int_{0}^{t}(t-s)^{\alpha-2} E_{\alpha-\beta, \alpha-1}\left(A(t-s)^{\alpha-\beta}\right)\left[f\left(s, x_{n}(s)\right)-f(s, x(s))\right] d s\right\| \\
& \leq b \sum_{i=1}^{k} d_{i}\left\|x_{n}^{\prime}\left(t_{i}^{-}\right)-x^{\prime}\left(t_{i}^{-}\right)\right\|+b L_{f} \int_{0}^{t}(t-s)^{\alpha-2}\left\|x_{n}(s)-x(s)\right\| d s \\
& \leq b \sum_{i=1}^{k} d_{i}\left\|x_{n}^{\prime}-x^{\prime}\right\|+b L_{f} \frac{T^{\alpha-1}}{\alpha-1}\left\|x_{n}-x\right\| .
\end{aligned}
$$


As $x_{n} \rightarrow x, x_{n}^{\prime} \rightarrow x^{\prime}$, it is easy to see that

$\left\|\left(F x_{n}\right)(t)-(F x)(t)\right\| \longrightarrow 0, \quad\left\|\left(F x_{n}\right)^{\prime}(t)-(F x)^{\prime}(t)\right\| \longrightarrow 0$, as $n \rightarrow \infty$.

Hence, $\left\|F x_{n}-F x\right\|_{P C^{1}} \rightarrow 0$, as $x_{n} \rightarrow x, x_{n}^{\prime} \rightarrow x^{\prime}$. Then $F$ is continuous on interval $B_{r}$.

Step 3: We prove $F$ is equicontinuous on $B_{r}$. Let $0 \leq t_{i} \leq \tau_{1}<\tau_{2} \leq$ $t_{i+1} \leq T$, then for each $x \in B_{r}$, we obtain:

$$
\begin{aligned}
& \left\|(F x)\left(\tau_{2}\right)-(F x)\left(\tau_{1}\right)\right\| \\
& \leq\left\|E_{\alpha-\beta}\left(A \tau_{2}^{\alpha-\beta}\right) x_{0}-E_{\alpha-\beta}\left(A \tau_{1}^{\alpha-\beta}\right) x_{0}\right\| \\
& +\left\|\tau_{2} E_{\alpha-\beta, 2}\left(A \tau_{2}^{\alpha-\beta}\right) x_{0}^{\prime}-\tau_{1} E_{\alpha-\beta, 2}\left(A \tau_{1}^{\alpha-\beta}\right) x_{0}^{\prime}\right\| \\
& +\left\|A \tau_{1}^{\alpha-\beta} E_{\alpha-\beta, \alpha-\beta+1}\left(A \tau_{1}^{\alpha-\beta}\right) x_{0}-A \tau_{2}^{\alpha-\beta} E_{\alpha-\beta, \alpha-\beta+1}\left(A \tau_{2}^{\alpha-\beta}\right) x_{0}\right\| \\
& +\sum_{j=1}^{i}\left\|E_{\alpha-\beta}\left(A\left(\tau_{2}-t_{j}\right)^{\alpha-\beta}\right)-E_{\alpha-\beta}\left(A\left(\tau_{1}-t_{j}\right)^{\alpha-\beta}\right)\right\|\left\|I_{j}\left(x\left(t_{j}^{-}\right)\right)\right\| \\
& +\sum_{j=1}^{i} \|\left(\tau_{2}-t_{j}\right) E_{\alpha-\beta, 2}\left(A\left(\tau_{2}-t_{j}\right)^{\alpha-\beta}\right) \\
& -\left(\tau_{1}-t_{j}\right) E_{\alpha-\beta, 2}\left(A\left(\tau_{1}-t_{j}\right)^{\alpha-\beta}\right)\|\| J_{j}\left(x^{\prime}\left(t_{j}^{-}\right)\right) \| \\
& +\sum_{j=1}^{i} \|\left(\tau_{1}-t_{j}\right)^{\alpha-\beta} A E_{\alpha-\beta, \alpha-\beta+1}\left(A\left(\tau_{1}-t_{j}\right)^{\alpha-\beta}\right) \\
& -\left(\tau_{2}-t_{j}\right)^{\alpha-\beta} A E_{\alpha-\beta, \alpha-\beta+1}\left(A\left(\tau_{2}-t_{j}\right)^{\alpha-\beta}\right)\|\| I_{j}\left(x\left(t_{j}^{-}\right)\right) \| \\
& +\| \int_{0}^{\tau_{2}}\left(\tau_{2}-s\right)^{\alpha-1} E_{\alpha-\beta, \alpha}\left(A\left(\tau_{2}-s\right)^{\alpha-\beta}\right)[B u(s)+f(s, x(s))] d s \\
& -\int_{0}^{\tau_{1}}\left(\tau_{1}-s\right)^{\alpha-1} E_{\alpha-\beta, \alpha}\left(A\left(\tau_{1}-s\right)^{\alpha-\beta}\right)[B u(s)+f(s, x(s))] d s \| \\
& \leq\left\|E_{\alpha-\beta}\left(A \tau_{2}^{\alpha-\beta}\right)-E_{\alpha-\beta}\left(A \tau_{1}^{\alpha-\beta}\right)\right\|\left\|x_{0}\right\| \\
& +\left\|\tau_{2} E_{\alpha-\beta, 2}\left(A \tau_{2}^{\alpha-\beta}\right)-\tau_{1} E_{\alpha-\beta, 2}\left(A \tau_{1}^{\alpha-\beta}\right)\right\|\left\|x_{0}^{\prime}\right\| \\
& +\left\|A \tau_{1}^{\alpha-\beta} E_{\alpha-\beta, \alpha-\beta+1}\left(A \tau_{1}^{\alpha-\beta}\right)-A \tau_{2}^{\alpha-\beta} E_{\alpha-\beta, \alpha-\beta+1}\left(A \tau_{2}^{\alpha-\beta}\right)\right\|\left\|x_{0}\right\| \\
& +\sum_{j=1}^{i}\left\|E_{\alpha-\beta}\left(A\left(\tau_{2}-t_{j}\right)^{\alpha-\beta}\right)-E_{\alpha-\beta}\left(A\left(\tau_{1}-t_{j}\right)^{\alpha-\beta}\right)\right\|
\end{aligned}
$$




$$
\begin{aligned}
& \times\left(h_{j}\left\|x\left(t_{j}^{-}\right)\right\|+\|I(0)\|\right)+\sum_{j=1}^{i} \|\left(\tau_{2}-t_{j}\right) E_{\alpha-\beta, 2}\left(A\left(\tau_{2}-t_{j}\right)^{\alpha-\beta}\right) \\
& -\left(\tau_{1}-t_{j}\right) E_{\alpha-\beta, 2}\left(A\left(\tau_{1}-t_{j}\right)^{\alpha-\beta}\right) \|\left(d_{j}\left\|x^{\prime}\left(t_{j}^{-}\right)\right\|+\|J(0)\|\right) \\
& +\sum_{j=1}^{i} A \|\left(\tau_{1}-t_{j}\right)^{\alpha-\beta} E_{\alpha-\beta, \alpha-\beta+1}\left(A\left(\tau_{1}-t_{j}\right)^{\alpha-\beta}\right) \\
& -\left(\tau_{2}-t_{j}\right)^{\alpha-\beta} E_{\alpha-\beta, \alpha-\beta+1}\left(A\left(\tau_{2}-t_{j}\right)^{\alpha-\beta}\right) \|\left(h_{j}\left\|x\left(t_{j}^{-}\right)\right\|+\|I(0)\|\right) \\
& +\int_{0}^{\tau_{1}} \|\left(\tau_{2}-s\right)^{\alpha-1} E_{\alpha-\beta, \alpha}\left(A\left(\tau_{2}-s\right)^{\alpha-\beta}\right) \\
& -\left(\tau_{1}-s\right)^{\alpha-1} E_{\alpha-\beta, \alpha}\left(A\left(\tau_{1}-s\right)^{\alpha-\beta}\right)\|\| f(s, x(s))+B u(s) \| d s \\
& +\left\|\int_{\tau_{1}}^{\tau_{2}}\left(\tau_{2}-s\right)^{\alpha-1} E_{\alpha-\beta, \alpha}\left(A\left(\tau_{2}-s\right)^{\alpha-\beta}\right)[f(s, x(s))+B u(s)] d s\right\| .
\end{aligned}
$$

By Hölder's inequality and $H(1)$ and $H(3)$, we obtain:

$$
\begin{aligned}
& \left\|(F x)\left(\tau_{2}\right)-(F x)\left(\tau_{1}\right)\right\| \\
& \leq\left\|E_{\alpha-\beta}\left(A \tau_{2}^{\alpha-\beta}\right)-E_{\alpha-\beta}\left(A \tau_{1}^{\alpha-\beta}\right)\right\|\left\|x_{0}\right\| \\
& \quad+\left\|\tau_{2} E_{\alpha-\beta, 2}\left(A \tau_{2}^{\alpha-\beta}\right)-\tau_{1} E_{\alpha-\beta, 2}\left(A \tau_{1}^{\alpha-\beta}\right)\right\|\left\|x_{0}^{\prime}\right\| \\
& \quad+\left\|A \tau_{1}^{\alpha-\beta} E_{\alpha-\beta, \alpha-\beta+1}\left(A \tau_{1}^{\alpha-\beta}\right)-A \tau_{2}^{\alpha-\beta} E_{\alpha-\beta, \alpha-\beta+1}\left(A \tau_{2}^{\alpha-\beta}\right)\right\|\left\|x_{0}\right\| \\
& \quad+\sum_{j=1}^{i}\left\|E_{\alpha-\beta}\left(A\left(\tau_{2}-t_{j}\right)^{\alpha-\beta}\right)-E_{\alpha-\beta}\left(A\left(\tau_{1}-t_{j}\right)^{\alpha-\beta}\right)\right\| \\
& \quad \times\left(h_{j}\left\|x\left(t_{j}^{-}\right)\right\|+\|I(0)\|\right)+\sum_{j=1}^{i} \|\left(\tau_{2}-t_{j}\right) E_{\alpha-\beta, 2}\left(A\left(\tau_{2}-t_{j}\right)^{\alpha-\beta}\right) \\
& \quad-\left(\tau_{1}-t_{j}\right) E_{\alpha-\beta, 2}\left(A\left(\tau_{1}-t_{j}\right)^{\alpha-\beta}\right) \|\left(d_{j}\left\|x^{\prime}\left(t_{j}^{-}\right)\right\|+\|J(0)\|\right) \\
& \quad+\sum_{j=1}^{i} \|\left(\tau_{1}-t_{j}\right)^{\alpha-\beta} A E_{\alpha-\beta, \alpha-\beta+1}\left(A\left(\tau_{1}-t_{j}\right)^{\alpha-\beta}\right) \\
& \quad-\left(\tau_{2}-t_{j}\right)^{\alpha-\beta} A E_{\alpha-\beta, \alpha-\beta+1}\left(A\left(\tau_{2}-t_{j}\right)^{\alpha-\beta}\right) \|\left(h_{j}\left\|x\left(t_{j}^{-}\right)\right\|+\|I(0)\|\right) \\
& \quad+\sup _{s \in\left[0, \tau_{1}\right]} \|\left(\tau_{2}-s\right)^{\alpha-1} E_{\alpha-\beta, \alpha}\left(A\left(\tau_{2}-s\right)^{\alpha-\beta}\right) \\
& \quad-\left(\tau_{1}-s\right)^{\alpha-1} E_{\alpha-\beta, \alpha}\left(A\left(\tau_{1}-s\right)^{\alpha-\beta}\right) \|\left(\|\Psi\|_{L^{1 / \gamma}} T^{1-\gamma}+\theta T r+M_{B} \varrho T\right) \\
& +b\|\Psi\|_{L^{1 / \gamma}}\left(\tau_{2}-\tau_{1}\right)^{1-\gamma}+b\left(\theta r+M_{B} \varrho\right)\left(\tau_{2}-\tau_{1}\right),
\end{aligned}
$$


where

$$
\begin{aligned}
\varrho:= & a_{6} \times\left[a_{7}+b k(2\|I(0)\|+\|J(0)\|)+b \sum_{i=1}^{k}\left(2 h_{i}+d_{i}\right) r\right. \\
& +b T^{1-\gamma}\|\Psi\|_{L^{1 / \gamma}}+b T \theta r .
\end{aligned}
$$

Similarly, we also have

$$
\begin{aligned}
& \left\|(F x)^{\prime}\left(\tau_{2}\right)-(F x)^{\prime}\left(\tau_{1}\right)\right\| \\
& \leq\left\|E_{\alpha-\beta}\left(A \tau_{2}^{\alpha-\beta}\right)-E_{\alpha-\beta}\left(A \tau_{1}^{\alpha-\beta}\right)\right\|\left\|x_{0}^{\prime}\right\|+\sum_{j=1}^{i} \| E_{\alpha-\beta}\left(A\left(\tau_{2}-t_{j}\right)^{\alpha-\beta}\right) \\
& \quad-E_{\alpha-\beta}\left(A\left(\tau_{1}-t_{j}\right)^{\alpha-\beta}\right) \|\left(d_{j}\left\|x^{\prime}\left(t_{j}^{-}\right)\right\|+\|J(0)\|\right) \\
& \quad+\sup _{s \in\left[0, \tau_{1}\right]} \|\left(\tau_{2}-s\right)^{\alpha-2} E_{\alpha-\beta, \alpha-1}\left(A\left(\tau_{2}-s\right)^{\alpha-\beta}\right) \\
& \quad-\left(\tau_{1}-s\right)^{\alpha-2} E_{\alpha-\beta, \alpha-1}\left(A\left(\tau_{1}-s\right)^{\alpha-\beta}\right) \| \\
& \quad\left(\|\Psi\|_{L^{1 / \gamma}} T^{1-\gamma}+T \theta r+M_{B} \varrho T\right)+\omega\left(\tau_{2}-\tau_{1}\right)^{\alpha-1-\gamma} \\
& +\frac{b\left(\theta r+M_{B} \varrho\right)}{\alpha-1}\left(\tau_{2}-\tau_{1}\right)^{\alpha-1},
\end{aligned}
$$

where

$$
\omega:=b\|\Psi\|_{L^{1 / \gamma}}\left(\frac{1-\gamma}{\alpha-1-\gamma}\right)^{1-\gamma}
$$

From the above arguments, let $\tau_{2} \rightarrow \tau_{1}$. Then we have

$$
\left\|(F x)\left(\tau_{2}\right)-(F x)\left(\tau_{1}\right)\right\| \longrightarrow 0,
$$

and

$$
\left\|(F x)^{\prime}\left(\tau_{2}\right)-(F x)^{\prime}\left(\tau_{1}\right)\right\| \longrightarrow 0 .
$$

Thus, $F$ is equicontinuous on interval $B_{r}$.

By Arzela-Ascoli's theorem, we obtain that the operator $F$ is completely continuous and by the Schauder fixed-point theorem, we obtain that $F$ has a fixed point $x$ on $B_{r}$. This further implies that system (1.2) has a solution $x$ in $P C^{1}\left(J, \mathbb{R}^{n}\right)$. Hence, system (1.2) is controllable on $J$. The proof is completed. 


\section{REFERENCES}

1. K. Balachandran, V. Govindaraj, M.D. Ortigueira, M. Reiver and J.J. Trujillo, Observability and controllability of fractional linear dynamical systems, in Sixth workshop on fractions and applications, France, 2013.

2. K. Balachandran, V. Govindaraj, M. Reiver and J.J. Trujillo, Controllability of fractional damped dynamical systems, Appl. Math. Comp. 257 (2015), 66-73.

3. A. Debbouche and D. Baleanu, Controllability of fractional evolution nonlocal impulsive quasilinear delay integro-differential systems, Comp. Math. Appl. 62 (2011), 1442-1450.

4. M. Feckan, Y. Zhou and J.R. Wang, On the concept and existence of solution for impulsive fractional differential equations, Comm. Nonlin. Sci. Numer. Simu. 17 (2012), 3050-3060.

5. - Response to Comments on the concept of existence of solution for impulsive fractional differential equations, Comm. Nonlin. Sci. Numer. Simu. 19 (2014), 4213-4215.

6. T.L. Guo, Controllability and observability of impulsive fractional linear time-invariant system, Comp. Math. Appl. 64 (2012), 3171-3182.

7. A.A. Kilbas, H.M. Srivastava and J.J. Trujillo, Theory and applications of fractional differential equation, North-Holland Math. Stud. 204, Elsevier Science, Amsterdam, 2006.

8. Z.H. Liu and B.J. Bin, Approximate controllability of impulsive RiemannLiouville fractional equations in Banach spaces, J. Int. Equat. Appl. 26 (2014), $527-551$.

9. Z.H. Liu and X.W. Li, Existence and uniqueness of solutions for the nonlinear impulsive fractional differential equations, Comm. Nonlin. Sci. Numer. Simu. 18 (2013), 1362-1373.

10. - On the exact controllability of impulsive fractional semilinear functional differential inclusions, Asian J. Contr. 17 (2015), 1857-1865.

11. _ Approximate controllability of fractional evolution systems with Riemann-Liouville fractional derivatives, SIAM J. Contr. Optim. 53 (2015), 19201933.

12. Approximate controllability for a class of hemivariational inequalities, Nonlin. Anal. Real World Appl. 22 (2015), 581-591.

13. K.X. Li and J.G. Peng, Laplace transform and fractional differential equations, Appl. Math. Lett. 24 (2011), 2019-2023.

14. F. Mainardi and R.Gorenflo, On Mittage Leffler type functions in fractional evolution process, J. Appl. Math. Comp. 118 (2000), 283-299.

15. I. Podlubny, Fractional differential equations, Academic Press, New York, 1993.

16. R. Sakthivel, N.I. Mahmudov and J.H. Kim, On controllability of second order nonlinear impulsive differential systems, Nonlinear Anal. TMA 71 (2009), $45-52$. 
17. Z.X. Tai and S. X. Lun, On controllability of fractional impulsive neutral infinite delay evolution integrodifferential systems in Banach spaces, Appl. Math. Lett. 25 (2012), 104-110.

18. V. Vijayakumar, C. Ravichandran, R. Murugesu and J.J. Trujillo, Controllability results for a class of fractional semilinear integro-differential inclusions via resolvent operators, Appl. Math. Comp. 247 (2014), 152-161.

19. G.T. Wang, B. Ahmad, L. Zhang and J.J.Nieto, Comments on the concept of existence of solution for impulsive fractional differential equations, Comm. Nonlin. Sci. Numer. Simu. 19 (2014), 401-403.

20. J.R. Wang, M. Feckan and Y. Zhou, On the concept of solution and existence results for impulsive fractional evolution equations, Dynam. Partial Diff. Eq. 8 (2011), 345-361.

21. C.E. Xiao, B. Zeng and Z.H. Liu, Feedback control for fractional impulsive evolution systems, Appl. Math. Comp. 268 (2015), 924-936.

22. T. Yang, Impulsive systems and control: Theory and applications, SpringerVerlag, Berlin, 2001.

23. Y. Zhou, Fractional evolution equations and inclusions: Analysis and control, Elsevier \& Academic Press, New York, 2016.

24. _ Basic theory of fractional differential equations, World Scientific, Singapore, 2014.

25. Y. Zhou and F. Jiao, Nonlcal Cauchy problem for fractional evolution equation, Nonlin. Anal. RWA 11 (2010), 4465-4475.

26. Y. Zhou, V. Vijayakumar and R. Murugesu, Controllability for fractional evolution inclusions without compactness, Evol. Eq. Contr. Theory 4 (2015), 507524 .

Guangxi Key Laboratory of Universities Optimization Control and Engineering Calculation, and College of Sciences, Guangxi University for Nationalities, Nanning 530006, Guangxi Province, P.R. China

Email address: zhhliu@hotmail.com

College of Sciences, Guangxi University for Nationalities, Nanning 530006, Guangxi Province, P.R. China

Email address: 578567208@qq.com 